\title{
Atheroembolic Renal Disease: A Beneficial Effect of Steroids
}

\section{Georges EI Hachem ${ }^{1^{*}}$ and Rim Al Nabbout ${ }^{2}$}

${ }^{1}$ Department of Medicine, Cardiology Division, St Georges Hospital University Medical Center, Dubai

${ }^{2}$ Department of Medicine, Internal Medicine Division, St Georges Hospital University Medical Center, Dubai

*Corresponding author: Georges El Hachem, St George Hospital, Ashrafieh Beirut, St Georges Hospital University Medical Center, Dubai, Tel: 6506189889; E-mail: georgesyelhachem@gmail.com

Received date: December 4, 2016; Accepted date: December 10, 2016; Published date: December 16, 2016

Copyright: (c) 2016 Hachem GE, et al. This is an open-access article distributed under the terms of the Creative Commons Attribution License, which permits unrestricted use, distribution, and reproduction in any medium, provided the original author and source are credited.

\begin{abstract}
Atheroembolic renal disease (AERD) or cholesterol crystal embolisation often is an under diagnosed clinical illness. It is a multisystemic disease with progressive renal failure due to foreign body reaction of cholesterol crystals flushed into a small vessel system of the kidneys from the arteriosclerotic plaques. It usually occurs after vascular catheterization, anticoagulation and less frequently spontaneously. Renal and patient prognosis are usually poor.

We hereby are reporting a case report of a 62-year-old male patient with dyslipidemia who underwent percutaneous coronary intervention for unstable angina and consecutive AERD with typical clinical appearance few days after the event. Rapid improvement of renal function was noticed after treatment with systemic corticosteroids.
\end{abstract}

Keywords: Atheroembolic renal disease (AERD); Prednisolone; Cardiac catheterization

\section{Introduction}

Atheroembolic renal disease (AERD) often is an underdiagnosed clinical illness. The kidney is usually involved because of the proximity of renal arteries to the abdominal aorta, where the erosion of atheromatous plaque most likely occurs. It is a multisystemic disease with progressive renal failure due to foreign body reaction of cholesterol crystals flushed into a small vessel system of the kidneys from the arteriosclerotic plaques. It usually occurs after vascular catheterization, anticoagulation and less frequently spontaneously. The classic triad of a precipitating event, acute or subacute renal failure, and skin lesions, are strongly suggestive of the disorder. Eosinophilia further supports the diagnosis, usually confirmed by biopsy of an affected organ or by the fundoscopic finding of cholesterol crystals in the retinal circulation. Renal and patient prognosis is usually poor.

Steroids might have a role in acute or subacute progressive forms with systemic inflammation and may result in rapid improvement of renal function.

The case reported is a 62-year-old male patient with dyslipidemia undergoing percutaneous coronary intervention after unstable angina and consecutive AERD with typical clinical appearance few days after the event.

\section{Case Presentation}

A 62-year-old male patient presented with recent recurrent episodes of severe oppressive chest pain. An electrocardiogram showed no ST elevations, T-troponin level was negative, and a chest X-ray was within normal limits.

Further co-morbidities consisted of hypercholesterolemia, smoking (30 pack/year). Last laboratory workup was done 6 months before presentation revealed a serum creatinine of $0.9 \mathrm{mg} / \mathrm{dl}$, serum cholesterol $188 \mathrm{mg} / \mathrm{dl}$, LDL-cholesterol $112 \mathrm{mg} / \mathrm{dl}$, HDL-cholesterol 29 $\mathrm{mg} / \mathrm{dl}$.

At admission, the serum creatinine was $1 \mathrm{mg} / \mathrm{dl}$. Daily home medications consisted of $100 \mathrm{mg}$ aspirin and $20 \mathrm{mg}$ atorvastatin.

The patient was admitted for coronary angiography. An $80 \%$ short stenosis at the Proximal segment of the Left Anterior Descending artery (LAD), 60\% stenosis of the second marginal, and 75\% stenosis of the proximal segment of the right coronary artery (RCA) was documented.

Cardiac ultrasound showed normal biventricular systolic and diastolic function without wall motion abnormalities as well as normal valvular function. $48 \mathrm{hrs}$ later, patient was discharged with a stable serum creatinin on a $\beta$ blocker, atorvastatin, aspirin, clopidogrel and an anti-anginal treatment, and scheduled to do PCI of the proximal LAD and proximal RCA with stent placement after few days.

Two days later, he developed one episode of upper GI bleeding. Gastroscopy was done showing multiple small ulcers in the stomach and duodenum in favor of ulcerative gastroduodenitis. Serum creatinine level was $2.4 \mathrm{mg} / \mathrm{dl}$. The main differential diagnosis of the acute kidney injury was either contrast-induced nephropathy v/s atheroembolic renal disease. Patient didn't require any blood transfusion. Esomeprazole was added to his medications, and PCI was held due to renal failure.

Two weeks after the coronary intervention, the patient was reassessed; serum creatinine level was $5.6 \mathrm{mg} / \mathrm{dl}$ (eGFR $16 \mathrm{~mL} / \mathrm{min} /$ $1.73 \mathrm{~m}^{2}$ ). Mild peripheral eosinophilias (889 cells/microl) with low C3 level $(88 \mathrm{mg} / \mathrm{dl})$ were also noted. The patient complained of muscle pain descending from the pelvic area in both legs. The skin demonstrated typical purple toes with livedo reticularis and incipient necrotic spots at different acral locations (Figure 1) although the distal pulses were palpable in both extremities. 


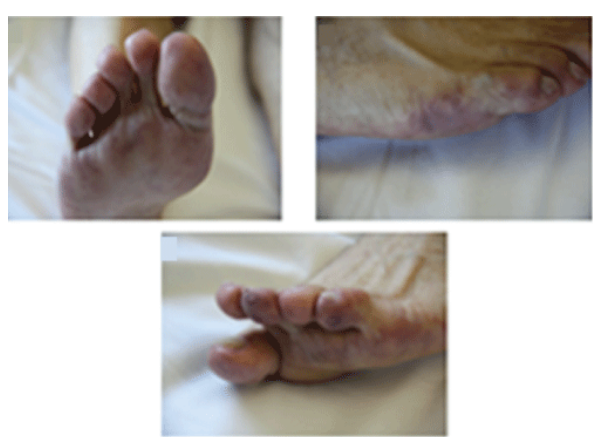

Figure 1: Blue toe syndrome of variable severity with livedo reticularis.

Skin biopsy (Figure 2) was done showing occlusion of the peripheral arterioles with biconvex, needle-shaped clefts appearing as "ghosts" which are characteristics of cholesterol crystal embolization.
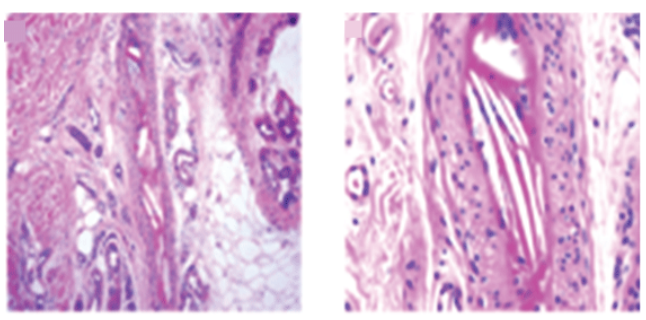

Figure 2: Skin biopsy with characteristics of cholesterol crystal embolization.

Considering the elapse in time between the intervention and the event, the progressive decline of renal function, peripheral eosinophilia, hypocomplementemia and the features of the skin biopsy, the presented was attributed to AERD.

Because of the continuous decline in kidney function, the patient was started on prednisolone $60 \mathrm{mg} /$ day for 4 weeks than tapered to 10 $\mathrm{mg} /$ day over one month. Kidney function improved to be stabilized with a serum creatinine level of $1.6 \mathrm{mg} / \mathrm{dl}$ and the patient was referred to $\mathrm{CABG}$ which was done without complications.

\section{Discussion}

Atheroembolic renal disease, sometimes referred to as renal cholesterol crystal embolization is an underdiagnosed cause of acute kidney injury. Etiologically, it is believed that renal arteries, small vessels, and glomerular capillaries are occluded with crystal material that originated from artheromatous plaques along abdominal aorta, spontaneously or during a coronary artery intervention or anticoagulation. Typically, embolization affects the kidneys, skin, brain, and gastrointestinal system. For this reason, atheroembolic renal disease is regarded as part of a multisystemic disorder [1].

Panum [2] first described atheroembolism in 1862, in the autopsy report of the Danish sculptor, Thorwaldsen, who died from a heart attack. In a coronary artery, a ruptured atheroma was identified, with atheromatous material filling the lumen distally. In English records, the first report of atheroembolism was made in 1926 by Benson [3] who reviewed three cases of coronary embolisation. 40 years later, Fine et al. [1] reviewed 221 cases of cholesterol crystal embolisation, underlining the low rate of antemortum clinical diagnosis. In the past two decades, atheroembolic renal disease has changed from being a pathological curiosity to a clinical syndrome. Thus, this disease can now be thought as a recognizable cause of renal disease, with diagnosis before death possible in most cases.

But to what extent can the diagnosis of artheroembolic renal disease be confirmed only by clinical features in a typical patient without performing a renal biopsy?

The exact incidence of artheroembolic renal disease is not known. The disorder predominantly affects men and white people, possibly because of decreased recognition of the cutaneous lesions in black population. In clinical series, prevalence varies, probably because of sampling bias. In non selected autopsy series [4], the frequency of atheroemboli findings range from $0.31 \%$ to $2.4 \%$. However, in autopsy studies [5] done in elderly patients and those who died after aortography or aortic surgery, researchers have reported an increased frequency, from $12 \%$ to $77 \%$. In two large renal biopsy studies [6] frequency of $1 \%$ was reported. However, the prevalence was higher in people older than age 60 years.

Clinical features demonstrate a large variation including neurologic, abdominal, cutaneous, and unspecific general symptoms besides other organ failure [1].

In one review of 221 patients, $34 \%$ of the patients show skin lesions as livedo reticularis, gangrene, or cyanosis1, and the incidence of renal function decrease is described to be between $30 \%$ and $40 \%$ [1]. In addition to acute or chronic loss of renal function, the patient presented herein showed typical signs of cholesterol embolism such as livedo reticularis, myalgias, and nail pulp infarction as well as necrosis in both lower extremities. Arterial feet pulses were palpable on both sides, which allow the distinction of lesions induced by AERD from peripheral artery disease. Because small vessels are affected in patients with AERD, necrosis coexists with palpable distal pulses because blood flow in these vessels is maintained through larger arteries [1].

Presence of eosinophilia should raise the level of suspicion. Renal biopsy is regarded as the definitive method for diagnosis. It yields a positive diagnosis in more than $75 \%$ of patients with acute or subacute renal failure. However, taking a renal biopsy sample is not always feasible, especially in sick patients. Taking a biopsy sample of skin lesions, a straightforward, and relatively non-invasive procedure, has a high diagnostic yield approaching 92\% [7]. The best sites for biopsy were noted to be feet and lower legs. Diagnosis by tissue sample is not needed in the presence of the classic triad (precipitating event, acute or subacute renal failure, and skin lesions), these patients can be diagnosed solely on a clinical basis [8].

The patient discussed did not undergo a renal biopsy because of the typical clinical picture and skin biopsy results. At the time of onset, he was diagnosed with coronary artery disease, heavy smoking, and dyslipidemia. Given the time between a cardiac event and the onset of renal failure, which evolved 2 weeks after clinical treatment, the diagnosis of AERD becomes more likely.

Atheroemboli are irregulary shaped and nondistensable crystal bodies and so they lead to an incomplete occlusion of small kidney vessels [9]. Over time, a foreign body reaction occurs with intimal proliferation and giant cell formation causing complement activation 
and inflammation, which further lead to progressive constriction of the vessel and decline of renal function. Considering the typical features such as livedo reticularis, myalgias, and blue toes [1] which the patient showed after hospitalization, the summary of preexisting risk factors, and the skin biopsy results, the diagnosis of AERD can be confirmed.

In addition to risk profile, the patient had undergone vascular catheterization, which is well known to be strongly associated with AERD [10]. A temporal relation to procedure may not be obvious. The exact incidence after invasive procedure is also not known because the data are relatively sparse and the published literature is mostly confined to single case reports. A review of the information suggests an estimated cumulative incidence of renal failure of $2 \%$ after an intravascular intervention. In the absence of renal biopsy, a definite distinction from contrast nephropathy is hard to make. Vascular intervention has shown to be close before onset of renal failure in $25 \%$ to $80 \%$ of patients with cholesterol embolization the patient described here underwent a coronary intervention 2 days before the onset of renal failure. As indicated above, an important differential diagnosis of renal failure besides AERD after a coronary intervention is contrast induced nephropathy.

The pathophysiology leading to the impairment of renal function is not exactly known. Tubular function decline due to intrarenal vascular constriction, rheological alteration caused by release of endothelin, adenosine, or reactive oxygen species have been proposed along with cytotoxic effects induced by the high osmolarity of ionic contrast agents themselves [11]. The patient discussed herein had been hospitalized due to concern of acute coronary syndrome and underwent a coronary intervention which was the only risk factor for development of contrast induced nephropathy. In contrast to AERD, CIN leads to acute impairment of renal function 24 to 48 hours after administration of contrast, and is generally reversible within 10 days.

Cholesterol embolism causes delayed loss of kidney function due to a foreign body reaction and is usually persistent or leads to prolonged worsening of renal insufficiency [10]. While patients with CIN mostly recover renal function, the outcome of AERD is poor. Data suggest that $30 \%$ of affected patients require renal replacement therapy within 2 to 6 months after the event; only $30 \%$ of these regain sufficient renal function to stop dialysis treatment. With the presence of heart failure, older age, underlying CKD or gastrointestinal and leg involvement, renal survival is additionally reduced. Overall patient survival correlates with renal outcome, and the most frequent cause of death includes cardiovascular events [11].

\section{Conclusion}

After consideration of risk factors, lapse of time, skin biopsy, and especially clinical appearance, the case reported was diagnosed with AERD.

Concerning the treatment, no clinical trials for patients with atheroembolic renal disease have been done. However, results of some studies of steroids suggest that they might have a potential role. Possible benefits of steroids have been reported in small series [12]. Belenfant and co-workers [13] used low-dose steroid $(0.3 \mathrm{mg} / \mathrm{kg})$ in 18 patients with relapsing disease who had improved symptoms and nutritional intake. Results of other series [14] have shown beneficial effects with high doses of steroids. Conversely, other reports4 have shown little or marginal benefit from steroid treatment. In a prospective study 9 of 354 patients with atheroembolic renal disease, steroids were not associated with improved renal or patient outcomes.

Our patient was started on prednisolone $0.8 \mathrm{mg} / \mathrm{kg} /$ day for 4 weeks because of the continuous decline of kidney function. Serum creatinine levels, which reached a peak of $5.6 \mathrm{mg} / \mathrm{dl} 2$ weeks after the coronary intervention, began to decline rapidly following the corticosteroid treatment. The clinical symptoms of myalgias, abdominal pain and eosinophilia subsided. Prednisolone was tapered to $10 \mathrm{mg} /$ day per OS over one month, serum creatinin level decreased and stabilized to $1.6 \mathrm{mg} / \mathrm{dl}$. No further increase in serum creatinine level was observed.

To our knowledge, the cholesterol embolisation case presented here is one of the rare cases showing rapid improvement in kidney function and avoidance of hemodialysis following oral corticosteroid administration. This fact may help us to suggest that corticosteroid treatment may be warranted in cholesterol embolism. A study with a larger number of patients may give more definitive directions for the use of corticosteroids as a treatment for cholesterol embolism.

\section{References}

1. Fine MJ, Kapoor W, Falanga V (1987) Cholesterol crystal embolization: A review of 221 cases in English literature. Angiology 38: 761-784.

2. Panum PL (1862) Experimentell Beitrage zur Lehre von der Embolie. Virchows Arch 25: 308-338.

3. Benson RL (1926) The present status of coronary arterial disease. Arch Pathol Lab Med 2: 876-916.

4. Cross S (1991) How common is cholesterol embolism? J Clin Pathol 44: 859-861.

5. Gore I, Collins D (1960) Spontaneous atheromatous embolization. Review of the literature and a report of 16 additional cases. Am J Clin Pathol 33: 416-426.

6. Jones DB, Iannaccone PM (1975) Atheromatous emboli in renal biopsies. An ultrastructural study. Am J Pathol 78: 261-276.

7. Frock J, Bierman M, Hammeke M, Reyes A (1994) Atheroembolic renal disease: experience with 22 patients. Nebr Med J 79: 317-321.

8. Scolari F, Ravani P, Gaggi R, Santostefano M, Rollino C, et al. The challenge of diagnosing atheroembolic renal disease: clinical features and prognostic factors. Circulation 116: 298-304.

9. Manesse CK, Blankestijn PJ, Man in' tVeld AJ, Schalekamp MA (1991) Renal failure and cholesterol crystal embolization: A report of 4 surviving cases and a review of literature. Clin Nephrol 36: 240-245.

10. Fukumoto Y, Tsutsui H, Tsuchihashi M, Masumoto A, Takeshita A (2003) The incidence and risk factors of cholesterol embolization syndrome, a complication of cardiac catheterization: A prospective study. J Am Coll Cardiol 42: 211-216.

11. Salomon R (2005) The role of osmolality in the incidence of contrastinduced nephropathy: A systematic review of angiographic contrast media in high risk patients. Kidney Int 68: 2256-2263.

12. Yucel A, Kart-Koseoglu H, Demirhan B, Ozdemir F (2006) Cholesterol crystal embolization mimicking vasculitis: success with corticosteroid and cyclophosphamide therapy in two cases. Rheumatol Int 26: 454-460.

13. Belenfant X, Meyrier A, Jacquot C (1999) Supportive treatment improves survival in multivisceral cholesterol crystal embolism. Am J Kidney Dis 33: 840-850.

14. Dahlberg P, Frecentese D, Cogbill T (2006) Cholesterol embolism: experience with 22 histologically proven cases. Surgery 105: 737-746. 